\title{
Oscillatory and Asymptotic Behavior of Third Order Half-linear Neutral Differential Equation with "Maxima"
}

\author{
R.Arul ${ }^{1}$, A.Ashok ${ }^{2}$ and G.Ayyappan ${ }^{3}$ \\ ${ }^{1,2}$ Department of Mathematics, Kandaswami Kandar's College, Velur - 638 182, Namakkal Dt., Tamil Nadu, \\ India \\ ${ }^{3}$ Department of Mathematics, Periyar University College of Arts and Science, Pappireddipatti - 636 905, Tamil \\ Nadu, India. \\ 1rarulkkc@gmail.com³ayyapmath@gmail.com
}

\section{Abstract}

In this paper we study the oscillation and asymptotic behavior of the third order neutral differential equation with "maxima" of the form

$$
\left(a(t)((x(t)+p(t) x(\tau(t))))^{\alpha}\right)+q(t) \max _{[\sigma(t), t]} x^{\alpha}(s)=0, t \geq t_{0},
$$

where $\alpha$ is the quotient of odd positive integers. Some examples are given to illustrate the main results.

\section{Mathematics Subject Classification : 34C10, 34K11}

Keywords and Phrases: Oscillation; asymptotic behavior; third order; half-linear neutral differential equation with "maxima".

\section{Introduction}

In this paper, we study the oscillation and asymptotic behavior of the third order neutral differential equation with "maxima" of the form

$$
\left(a(t)((x(t)+p(t) x(\tau(t))))^{\alpha}\right)+q(t) \max _{[\sigma(t), t]} x^{\alpha}(s)=0, t \geq t_{0},
$$

where $\alpha$ is the quotient of odd positive integers, subject to the following conditions:

(H1) $a(t), p(t), q(t), \tau(t)$, and $\sigma(t) \in C\left(\left[t_{0}, \infty\right)\right)$;

(H2) $a(t)>0$, and $\int_{t_{0}}^{\infty} \frac{1}{a^{1 / \alpha}(t)} d t=\infty$;

(H3) $0 \leq p(t) \leq p<1, q(t) \geq 0$ and $q(t)$ is not identically zero on any ray of $q(t) \in\left[t_{x}, \infty\right)$ for any $t_{x} \geq t_{0}$;

(H4) $\tau(t) \leq t, \tau(t) \geq \tau_{0}>0, \sigma \circ \tau=\tau \circ \sigma$ with $\sigma(\tau(t)) \leq t$ and $\lim _{t \rightarrow \infty} \tau(t)=\lim _{t \rightarrow \infty} \sigma(t)=\infty$.

Set $z(t)=x(t)+p(t) x(\tau(t))$. By a solution of equation (1.1), we mean a continuous function $x(t) \in C\left(\left[T_{x}, \infty\right)\right), T_{x} \geq t_{0}$, which has the properties $z(t) \in C^{2}\left(\left[T_{x}, \infty\right)\right), a(t)\left(z^{\prime \prime}(t)\right)^{\alpha} \in C^{1}\left(\left[T_{x}, \infty\right)\right)$ and satisfies equation (1.1) on $\left[T_{x}, \infty\right)$. We consider only those solutions $x(t)$ of equation (1.1) which satisfy $\sup \{|x(t)|: t \geq T\}>0$ for all $T \geq t_{0}$. We assume that equation (1.1) possesses such a solution. A solution of equation (1.1) is called oscillatory if it has infinitely many zeros on $\left[T_{x}, \infty\right)$ and called nonoscillatory otherwise.

In recent years there has been great attention denoted to the oscillatory and asymptotic behavior of third order neutral differential equations without maxima, see $[1,7,8,9,13,14]$, and the references contained therein. However few results are available on the oscillatory behavior of third order differential equation with "maxima", see [2, 3, 4, 5, 6].

Motivated by these observations, in this paper, we present several sufficient conditions for the oscillatory behavior of solutions of equation (1.1). The results obtained in this paper extend that of in $[1,8,12,13,14]$ for the equation without maxima.

In Section 2, we present preliminary lemmas which will be used to prove that main results. Section 3 deals with oscillation results and in Section 4, we provide some examples to illustrate the main results. 


\section{Preliminary Lemmas}

In this section we present some useful lemmas, which will be used to prove the main results.

Lemma 2.1 Let $a, b \in[0, \infty)$. Then

$$
a^{\alpha}+b^{\alpha} \geq \frac{1}{2^{\alpha-1}}(a+b)^{\alpha}, \alpha \geq 1
$$

and

$$
a^{\alpha}+b^{\alpha} \geq(a+b)^{\alpha}, 0<\alpha \leq 1 \text {. }
$$

Proof. The proof can be found in [13].

Lemma 2.2 Let $f$ and $g \in C\left(\left[t_{0}, \infty\right), \mathrm{R}\right)$ and $\delta \in C\left(\left[t_{0}, \infty\right), \mathrm{R}\right)$ satisfies $\lim _{t \rightarrow \infty} \delta(t)=\infty$ and $\delta(t) \leq t$ for all $t \in\left[t_{0}, \infty\right)$; further suppose that there exists $h \in C\left(\left[t_{-1}, \infty\right), \mathrm{R}^{+}\right)$where $t_{-1}=\min _{t \in\left[t_{0}, \infty\right)}\{\delta(t)\}$, such that $f(t)=g(t) h(\delta(t))$ holds for all $t \in\left[t_{0}, \infty\right)$. Suppose that $\lim _{t \rightarrow \infty} f(t)$ exists and $\lim _{t \rightarrow \infty} \inf g(t)>-1$. Then $\lim _{t \rightarrow \infty} \operatorname{suph}(t)>0$ implies $\lim _{t \rightarrow \infty} f(t)>0$.

Proof. The proof can be found in [6].

Lemma 2.3 Let $x(t)$ be a positive solution of equation (1.1), then the corresponding function $z(t)$ satisfies one of the following two cases:

(l) $z(t)>0, z^{\prime}(t)>0, z^{\prime \prime}(t)>0,\left(a(t)\left(z^{\prime \prime}(t)\right)^{\alpha}\right)<0$;

(II) $z(t)>0, z^{\prime}(t)<0, z^{\prime \prime}(t)>0,\left(a(t)\left(z^{\prime \prime}(t)\right)^{\alpha}\right)<0$,

for all $t \geq T$, where $T$ is sufficiently large.

Proof. The proof can be found in [2], and hence omitted.

Lemma 2.4 Let $x(t)$ be a negative solution of equation (1.1), then the corresponding function $z(t)$ satisfies one of the following two cases:

(l) $z(t)<0, z^{\prime}(t)<0, z^{\prime \prime}(t)<0,\left(a(t)\left(z^{\prime \prime}(t)\right)^{\alpha}\right)>0$;

(II) $z(t)<0, z^{\prime}(t)>0, z^{\prime \prime}(t)<0,\left(a(t)\left(z^{\prime \prime}(t)\right)^{\alpha}\right)>0$,

for all $t \geq T$, where $T$ is sufficiently large.

The proof of Lemma 2.4 is analogous to that of Lemma 2.3.

Lemma 2.5 The function $x(t)$ is a negative solution of equation (1.1) if and only if $-x(t)$ is a positive solution of the equation

$$
\left(a(t)\left(z^{\prime \prime}(t)\right)^{\alpha}\right)+q(t) \min _{[\sigma(t), t]} x^{\alpha}(s)=0 .
$$

Proof. The assertion can be verified easily and hence omitted.

Lemma 2.6 Let $x(t)$ be positive function defined for all $t \geq t_{0}$. Then

$$
\max _{[\sigma(t), t]} x^{\alpha}(s)+\max _{[\sigma(\tau(t)), t]} x^{\alpha}(s) \geq \max _{[\sigma(t), t]}\left(x^{\alpha}(s)+x^{\alpha}(\tau(s))\right) .
$$

Proof. The proof can be found in [11].

Lemma 2.7 Let $x(t)$ be a positive solution of equation (1.1), and let the corresponding function $z(t)$ satisfy Case (II) of Lemma 2.3. If 


$$
\int_{t_{0}}^{\infty} \int_{s}^{\infty}\left(\frac{1}{a(\tau(u))} \int_{u}^{\infty} Q(v) d v\right)^{\frac{1}{\alpha}} d s d t=\infty
$$

where

$$
Q(t)=\min \{q(t), q(\tau(t))\}
$$

then $\lim _{t \rightarrow \infty} x(t)=\lim _{t \rightarrow \infty} z(t)=0$.

Proof. Let $x(t)$ be a positive solution of equation (1.1). We may only prove the case when $\alpha \geq 1$, since the case when $0<\alpha \leq 1$ is similar. From equation (1.1) and Case(II) of Lemma 2.3, we obtain

$$
\left(a(t)\left(z^{\prime \prime}(t)\right)^{\alpha}\right)+p_{0}^{\alpha} \frac{\left[\sigma(\tau(t))\left(z^{\prime \prime}(\tau(t))\right)^{\alpha}\right]^{\prime}}{\tau_{0}}+q(t) \max _{[\sigma(t), t]} x^{\alpha}(s)+p_{0}^{\alpha} q(\tau(t)) \max _{[\sigma(\tau(t)), \tau(t)]} x^{\alpha}(s) \leq 0 .
$$

which follows from (2.1) and (2.6), $\sigma \circ \tau=\tau \circ \sigma$ and Lemma 2.6 that

$$
\left(a(t)\left(z^{\prime \prime}(t)\right)^{\alpha}\right)+\frac{p_{0}^{\alpha}}{\tau_{0}}\left(a(\tau(t))\left(z^{\prime \prime}(\tau(t))\right)^{\alpha}\right)+\frac{Q(t)}{2^{\alpha-1}} \max _{[\sigma(t), t]} z^{\alpha}(s) \leq 0 .
$$

Since $z(t)$ is positive and decreasing, we obtain

$$
\left(a(t)\left(z^{\prime \prime}(t)\right)^{\alpha}\right)+\frac{p_{0}^{\alpha}}{\tau_{0}}\left(a(\tau(t))\left(z^{\prime \prime}(\tau(t))\right)^{\alpha}\right)+\frac{Q(t)}{2^{\alpha-1}} z^{\alpha}(\sigma(t)) \leq 0 .
$$

Integrating the last inequality from $t$ to $\infty$, we obtain

$$
a(t)\left(z^{\prime \prime}(t)\right)^{\alpha}+\frac{p_{0}^{\alpha}}{\tau_{0}} a(\tau(t))\left(z^{\prime \prime}(\tau(t))\right)^{\alpha} \geq \frac{1}{2^{\alpha-1}} \int_{t}^{\infty} Q(s) z^{\alpha}(\sigma(s)) d s .
$$

Since $\tau(t) \leq t$, and $a(t)\left(z^{\prime \prime}(t)\right)^{\alpha}$ is decreasing, we see that

$$
a(t)\left(z^{\prime \prime}(t)\right)^{\alpha} \leq a(\tau(t))\left(z^{\prime \prime}(\tau(t))\right)^{\alpha} .
$$

Thus

$$
a(\tau(t))\left(z^{\prime \prime}(\tau(t))\right)^{\alpha} \geq \frac{1}{2^{\alpha-1}\left(1+\frac{p_{0}^{\alpha}}{\tau_{0}}\right)} \int_{t}^{\infty} Q(s) z^{\alpha}(\sigma(s)) d s
$$

Inview of Case(II) of Lemma 2.3, we have $\lim _{t \rightarrow \infty} z(t)=L \geq 0$. Assume $L>0$, then $z^{\alpha}(\sigma(t)) \geq L^{\alpha}$. Then, we have

$$
z^{\prime \prime}(\tau(t)) \geq L\left(\frac{1}{2^{\alpha-1}\left(1+\frac{p_{0}^{\alpha}}{\tau_{0}}\right)}\right)^{\frac{1}{\alpha}}\left(\frac{1}{a(\tau(t))} \int_{t}^{\infty} Q(s) d s\right)^{\frac{1}{\alpha}} .
$$

Integrating the last inequality from $t$ to $\infty$, and then integrating the resulting inequality from $t_{1}$ to $\infty$, we obtain 


$$
\frac{1}{\tau_{0}^{2}} z\left(\tau\left(t_{1}\right)\right) \geq L\left(\frac{1}{2^{\alpha-1}\left(1+\frac{p_{0}^{\alpha}}{\tau_{0}}\right)}\right)^{\frac{1}{\alpha}} \int_{t_{1}}^{\infty} \int_{s}^{\infty}\left(\frac{1}{a(\tau(u))} \int_{u}^{\infty} Q(v) d v\right)^{\frac{1}{\alpha}} d u d s
$$

which contradicts (2.5). Thus $\lim _{t \rightarrow \infty} z(t)=0$. Since $\lim _{t \rightarrow \infty} x(t) \leq \lim _{t \rightarrow \infty} z(t)$ implies $\lim _{t \rightarrow \infty} x(t)=0$. This completes the proof.

Lemma 2.8 Assume that $z(t)$ satisfies Case (I) of Lemma 2.3 for $t \geq t_{1}$. Then

$$
z^{\prime}(t) \geq\left(a^{1 / \alpha} z^{\prime \prime}(t)\right) \beta_{1}\left(t, t_{1}\right)
$$

and

$$
z(t) \geq\left(a^{1 / \alpha}(t) z^{\prime \prime}(t)\right) \beta_{2}\left(t, t_{1}\right)
$$

where $\beta_{1}\left(t, t_{1}\right)=\int_{t_{1}}^{t} \frac{1}{a^{1 / \alpha}(s)} d s$ and $\beta_{2}\left(t, t_{1}\right)=\int_{t_{1}}^{t} \int_{t_{1}}^{s} \frac{1}{a^{1 / \alpha}(u)} d u d s$.

Proof. The proof can be found in [13].

\section{Oscillation Results}

In this section, we present sufficient conditions for the oscillatory behavior of solutions of equation (1.1). Throughout this section, without loss of generality we can deal only with the positive solution of equation (1.1) since the proof for the negative case is similar.

Theorem 3.1 Let $\alpha \geq 1$. If condition (2.5) holds, and assume that there exists a positive nondecreasing differentiable function $\rho(t)$ for $t \geq t_{2} \geq t_{1}$ such that

$$
\lim _{t \rightarrow \infty} \sup \int_{t_{2}}^{t}\left[\frac{\rho(s) Q(s)}{2^{\alpha-1}}-\frac{1}{(\alpha+1)^{\alpha+1}} \frac{\left(\rho^{\prime}(s)\right)^{\alpha+1}}{(\rho(s))^{\alpha}}\left(\frac{1}{\left(\beta_{1}\left(s, t_{1}\right)\right)^{\alpha}}+\frac{p_{0}^{\alpha}}{\tau_{0}} \frac{1}{\left(\beta_{1}\left(\tau(s), t_{1}\right) \tau^{\prime}(s)\right)^{\alpha}}\right)\right] d s=\infty,
$$

then every solution of equation (1.1) is either oscillatory or tends to zero as $t \rightarrow \infty$.

Proof. Let $x(t)$ be a positive solution of equation (1.1). Without loss of generality assume that $x(t)>0, x(\tau(t))>0$, and $x(\sigma(t))>0$ for all $t \geq t_{1} \geq t_{0}$. Then the corresponding function $z(t)$ satisfies Case (I) and Case (II) of Lemma 2.3 for all $t \geq t_{1}$.

Case(I). From the proof of Lemma 2.7, we have (2.7). Since $z(t)$ is positive and increasing, we have from (2.7) that

$$
\left(a(t)\left(z^{\prime \prime}(t)\right)^{\alpha}\right)+\frac{p_{0}^{\alpha}}{\tau_{0}}\left(a(\tau(t))\left(z^{\prime \prime}(\tau(t))\right)^{\alpha}\right)+Q(t) z^{\alpha}(t) \leq 0 .
$$

Define

$$
w(t)=\rho(t) \frac{a(t)\left(z^{\prime \prime}(t)\right)^{\alpha}}{z^{\alpha}(t)}, t \geq t_{1} .
$$

Then $w(t)>0$, and

$$
w^{\prime}(t)=\rho^{\prime}(t) \frac{a(t)\left(z^{\prime \prime}(t)\right)^{\alpha}}{z^{\alpha}(t)}+\rho(t) \frac{\left(a(t)\left(z^{\prime \prime}(t)\right)^{\alpha}\right)^{\prime}}{z^{\alpha}(t)}-\frac{\alpha \rho(t) a(t)\left(z^{\prime \prime}(t)\right)^{\alpha}}{z^{\alpha+1}(t)} z^{\prime}(t)
$$




$$
\leq \frac{\rho^{\prime}(t)}{\rho(t)} w(t)+\rho(t) \frac{\left(a(t)\left(z^{\prime \prime}(t)\right)^{\alpha}\right)^{\prime}}{z^{\alpha}(t)}-\frac{\alpha \beta_{1}\left(t, t_{1}\right)}{\rho^{1 / \alpha}(t)} w^{1+\frac{1}{\alpha}}(t) .
$$

Similarly, define another function $v$ by

$$
v(t)=\rho(t) \frac{a(\tau(t))\left(z^{\prime \prime}(\tau(t))\right)^{\alpha}}{z^{\alpha}(\tau(t))}, t \geq t_{1} .
$$

Then $v(t)>0$, and

$$
\begin{aligned}
v^{\prime}(t) & =\rho^{\prime}(t) \frac{a(\tau(t))\left(z^{\prime \prime}(\tau(t))\right)^{\alpha}}{z^{\alpha}(\tau(t))}+\rho(t) \frac{\left(a(\tau(t))\left(z^{\prime \prime}(\tau(t))\right)^{\alpha}\right)^{\prime}}{z^{\alpha}(\tau(t))}-\frac{\alpha \rho(t) a(\tau(t))\left(z^{\prime \prime}(\tau(t))\right)^{\alpha}}{z^{\alpha+1}(\tau(t))} z^{\prime}(\tau(t)) \tau^{\prime}(t) \\
& \leq \frac{\rho^{\prime}(t)}{\rho(t)} v(t)-\frac{\alpha \beta_{1}\left(\tau(t), t_{1}\right)}{\rho^{1 / \alpha}(t)} \tau^{\prime}(t) v^{1+\frac{1}{\alpha}}(t)+\rho(t) \frac{\left(a(\tau(t))\left(z^{\prime \prime}(\tau(t))\right)^{\alpha}\right)^{\prime}}{z^{\alpha}(\tau(t))} .
\end{aligned}
$$

From (3.4) and (3.6), we obtain

$$
\begin{gathered}
w^{\prime}(t)+\frac{p_{0}^{\alpha}}{\tau_{0}} v^{\prime}(t) \leq \frac{\rho(t)\left[a(t)\left(z^{\prime \prime}(t)\right)^{\alpha}\right]+\frac{p_{0}^{\alpha}}{\tau_{0}}\left[a(\tau(t))\left(z^{\prime \prime}(\tau(t))\right)^{\alpha}\right]}{z^{\alpha}(t)}+\frac{\rho^{\prime}(t)}{\rho(t)} w(t)-\frac{\alpha \beta_{1}\left(t, t_{1}\right)}{\rho^{1 / \alpha}(t)} w^{1+\frac{1}{\alpha}}(t) \\
+\frac{p_{0}^{\alpha}}{\tau_{0}}\left[\frac{\rho^{\prime}(t)}{\rho(t)} v(t)-\frac{\alpha \beta_{1}\left(\tau(t), t_{1}\right)}{\rho^{1 / \alpha}(t)} \tau^{\prime}(t) v^{1+\frac{1}{\alpha}}(t)\right]
\end{gathered}
$$

where we have used $\left(a(t)\left(z^{\prime \prime}(t)\right)^{\alpha}\right)^{\prime}$ is negative and $z(t)$ is increasing. Since $z(t)$ is increasing from (2.7) and (??), we obtain

$$
\begin{gathered}
w^{\prime}(t)+\frac{p_{0}^{\alpha}}{\tau_{0}} v^{\prime}(t) \leq-\frac{\rho(t) Q(t)}{2^{\alpha-1}}+\frac{\rho^{\prime}(t)}{\rho(t)} w(t)-\frac{\alpha \beta_{1}\left(t, t_{1}\right)}{\rho^{1 / \alpha}(t)} w^{1+\frac{1}{\alpha}}(t) \\
+\frac{p_{0}^{\alpha}}{\tau_{0}}\left[\frac{\rho^{\prime}(t)}{\rho(t)} v(t)-\frac{\alpha \beta_{1}\left(\tau(t), t_{1}\right)}{\rho^{1 / \alpha}(t)} \tau^{\prime}(t) v^{1+\frac{1}{\alpha}}(t)\right] .
\end{gathered}
$$

Now using (3.8) and the inequality

$$
B u-A u^{1+1 / \alpha} \leq \frac{\alpha^{\alpha}}{(\alpha+1)^{\alpha+1}} \frac{B^{\alpha+1}}{A^{\alpha}}, A>0
$$

we have

$w^{\prime}(t)+\frac{p_{0}^{\alpha}}{\tau_{0}} v^{\prime}(t) \leq-\frac{\rho(t) Q(t)}{2^{\alpha-1}}+\frac{1}{(\alpha+1)^{\alpha+1}} \frac{\left(\rho^{\prime}(t)\right)^{\alpha+1}}{\left(\rho(t) \beta_{1}\left(t, t_{1}\right)\right)^{\alpha}}+\frac{p_{0}^{\alpha}}{\tau_{0}(\alpha+1)^{\alpha+1}} \frac{\left(\rho^{\prime}(t)\right)^{\alpha+1}}{\left(\rho(t) \beta_{1}\left(\tau(t), t_{1}\right) \tau^{\prime}(t)\right)^{\alpha}}$

Integrating (3.10) from $t_{2}\left(t_{2} \geq t_{1}\right)$ to $t$, we get

$$
\int_{t_{2}}^{t}\left[\rho(s) \frac{Q(s)}{2^{\alpha-1}}-\frac{1}{(\alpha+1)^{\alpha+1}} \frac{\left(\rho^{\prime}(s)\right)^{\alpha+1}}{(\rho(s))^{\alpha}}\left(\frac{1}{\left(\beta_{1}\left(s, t_{1}\right)\right)^{\alpha}}+\frac{p_{0}^{\alpha}}{\tau_{0}} \frac{1}{\left(\beta_{1}\left(\tau(s), t_{1}\right) \tau^{\prime}(s)\right)^{\alpha}}\right)\right] d s \leq w\left(t_{2}\right)+\frac{p_{0}^{\alpha}}{\tau_{0}} v\left(t_{2}\right),
$$

which contradicts (3.1).

Case(II). From condition (2.5) and the proof of Lemma 2.7, we see that $\lim _{t \rightarrow \infty} x(t)=0$. This completes the proof of the theorem.

By Lemma 2.1, similar to the proof of Theorem 3.1, we have the following theorem. 
Theorem 3.2 Let $0<\alpha \leq 1$. If condition (2.5) holds, and assume that there exists a positive nondecreasing differentiable function $\rho(t)$ for $t \geq t_{2} \geq t_{1}$ such that

$$
\lim _{t \rightarrow \infty} \sup \int_{t_{2}}^{t}\left[\rho(s) Q(s)-\frac{1}{(\alpha+1)^{\alpha+1}} \frac{\left(\rho^{\prime}(s)\right)^{\alpha+1}}{(\rho(s))^{\alpha}}\left(\frac{1}{\left(\beta_{1}\left(s, t_{1}\right)\right)^{\alpha}}+\frac{p_{0}^{\alpha}}{\tau_{0}} \frac{1}{\left(\beta_{1}\left(\tau(s), t_{1}\right) \tau^{\prime}(s)\right)^{\alpha}}\right)\right] d s=\infty,
$$

then every solution of equation (1.1) is either oscillatory or tends to zero as $t \rightarrow \infty$.

Theorem 3.3 Let $\alpha \geq 1$. If condition (2.5) holds, and assume that there exists a positive nondecreasing differentiable function $\rho(t)$ for $t \geq t_{2} \geq t_{1}$ such that

$$
\lim _{t \rightarrow \infty} \sup \int_{t_{2}}^{t}\left[\frac{\rho(s) Q(s)}{2^{\alpha-1}}-\frac{\left(\rho^{\prime}(s)\right)^{2}}{4 \alpha \rho(s)}\left(\frac{\left(\beta_{2}\left(s, t_{1}\right)\right)^{1-\alpha}}{\beta_{1}\left(s, t_{1}\right)}+\frac{p_{0}^{\alpha}}{\tau_{0}} \frac{\left(\beta_{2}\left(\tau(s), t_{1}\right)\right)^{1-\alpha}}{\beta_{1}\left(\tau(s), t_{1}\right) \tau^{\prime}(s)}\right)\right] d s=\infty,
$$

then every solution of equation (1.1) is either oscillatory or tends to zero as $t \rightarrow \infty$.

Proof. Let $x(t)$ be a positive solution of equation (1.1). Without loss of generality assume that $x(t)>0, x(\tau(t))>0$ , and $x(\sigma(t))>0$ for all $t \geq t_{1} \geq t_{0}$. Then the corresponding function $z(t)$ satisfies Case (I) and Case (II) of Lemma 2.3 for all $t \geq t_{1}$.

Case(I). From the proof of Lemma 2.7, we have (2.7). From Lemma 2.8, we get (2.8) and (2.9).

Define the function $w$ and $v$ by (3.3) and (3.5), respectively. Proceeding as in the proof of Theorem 3.1, we have (3.4) and (3.6). It follows from (2.8) and (3.3), we get

$$
w^{1+\frac{1}{\alpha}}(t) \leq \frac{\beta_{2}^{\alpha-1}\left(t, t_{1}\right)}{\rho^{1-\frac{1}{\alpha}}(t)} w^{2}, t \geq t_{1}
$$

Using (3.13) in (3.4), we obtain

$$
w^{\prime}(t) \leq \frac{\rho(t)\left(a(t)\left(z^{\prime \prime}(t)\right)^{\alpha}\right)^{\prime}}{z^{\alpha}(t)}+\frac{\rho^{\prime}(t)}{\rho(t)} w(t)-\frac{\alpha\left(\beta_{2}\left(t, t_{n}\right)\right)^{\alpha-1}}{\rho(t)} \beta_{1}\left(t, t_{1}\right) w^{2}(t) .
$$

Similarly from (2.9) and (3.5), we get

$$
v^{1+\frac{1}{\alpha}}(t) \leq \frac{\left(\beta_{2}(\tau(t), t)\right)^{\alpha-1}}{\rho^{1-\frac{1}{\alpha}}(t)} v^{2}(t), t \geq t_{1}
$$

Using (3.15) in (3.6), we have

$$
v^{\prime}(t) \leq \frac{\rho(t)\left(a(\tau(t))\left(z^{\prime \prime}(\tau(t))\right)^{\alpha}\right)^{\prime}}{z^{\alpha}(\tau(t))}+\frac{\rho^{\prime}(t)}{\rho(t)} v(t)-\frac{\alpha\left(\beta_{2}\left(\tau(t), t_{1}\right)\right)^{\alpha-1} \beta_{1}\left(\tau(t), t_{1}\right) \tau^{\prime}(t)}{\rho(t)} v^{2}(t) .
$$

From (3.14) and (3.16), we obtain

$$
\begin{aligned}
w^{\prime}(t)+\frac{p_{0}^{\alpha}}{\tau_{0}} v^{\prime}(t) \leq \rho(t) \frac{\left[a(t)\left(z^{\prime \prime}(t)\right)^{\alpha}\right]+\frac{p_{0}^{\alpha}}{\tau_{0}}\left[a(\tau(t))\left(z^{\prime \prime}(\tau(t))\right)^{\alpha}\right]}{z^{\alpha}(t)} & \\
+ & \frac{\rho^{\prime}(t)}{\rho(t)} w(t)-\frac{\alpha\left(\beta_{2}\left(t, t_{1}\right)\right)^{\alpha-1} \beta_{1}\left(t, t_{1}\right)}{\rho(t)} w^{2}(t) \\
+ & \frac{p_{0}^{\alpha}}{\tau_{0}}\left[\frac{\rho^{\prime}(t)}{\rho(t)} v(t)-\frac{\alpha\left(\beta_{2}\left(\tau(t), t_{1}\right)\right)^{\alpha-1} \beta_{1}\left(\tau(t), t_{1}\right)}{\rho(t)} \tau^{\prime}(t) v^{2}(t)\right]
\end{aligned}
$$


where we have used $\tau(t) \leq t, z(t)$ is increasing and $\left(a(t)\left(z^{\prime \prime}(t)\right)^{\alpha}\right)^{\prime}$ is negative. Since $z(t)$ is positive and increasing, we have from (2.7) and (3.17), we obtain

$$
w^{\prime}(t)+\frac{p_{0}^{\alpha}}{\tau_{0}} v^{\prime}(t) \leq-\frac{\rho(t) Q(t)}{2^{\alpha-1}}+\frac{\left(\rho^{\prime}(t)\right)^{2}}{\alpha \rho(t)}\left[\frac{\left(\beta_{2}\left(t, t_{1}\right)\right)^{1-\alpha}}{\beta_{1}\left(t, t_{1}\right)}+\frac{p_{0}^{\alpha}}{\tau_{0}} \frac{\left(\beta_{2}\left(\tau(t), t_{1}\right)\right)^{1-\alpha}}{\beta_{1}\left(\tau(t), t_{1}\right) \tau^{\prime}(t)}\right]
$$

where we have used the inequality $B u-A u^{2} \leq \frac{B^{2}}{4 A}, A>0$. Integrating the above inequality from $t_{2}\left(t_{2} \geq t_{1}\right)$ to $t$, we obtain

$$
\int_{t_{2}}^{t}\left[\frac{\rho(s) Q(s)}{2^{\alpha-1}}-\frac{\left(\rho^{\prime}(s)\right)^{2}}{4 \alpha \rho(s)}\left(\frac{\left(\beta_{2}\left(s, t_{1}\right)\right)^{1-\alpha}}{\beta_{1}\left(s, t_{1}\right)}+\frac{p_{0}^{\alpha}}{\tau_{0}} \frac{\left(\beta_{2}\left(\tau(s), t_{1}\right)\right)^{1-\alpha}}{\beta_{1}\left(\tau(s), t_{1}\right) \tau^{\prime}(s)}\right)\right] d s \leq w\left(t_{2}\right)+\frac{p_{0}^{\alpha}}{\tau_{0}} v\left(t_{2}\right),
$$

which contradicts (3.12).

Case(II). The proof is similar to that of Case (II) of Theorem 3.1. The proof is now complete.

From (2.2), similar to that of proof of Theorem 3.3, we obtain the following results.

Theorem 3.4 Let $0<\alpha \leq 1$. If condition (2.5) holds, and assume that there exists a positive, nondecreasing differentiable function $\rho(t)$ for $t \geq t_{2} \geq t_{1}$ such that

$$
\lim _{t \rightarrow \infty} \sup \int_{t_{2}}^{t}\left[\rho(s) Q(s)-\frac{\left(\rho^{\prime}(s)\right)^{2}}{4 \alpha \rho(s)}\left(\frac{\left(\beta_{2}\left(s, t_{1}\right)\right)^{1-\alpha}}{\beta_{1}\left(s, t_{1}\right)}+\frac{p_{0}^{\alpha}}{\tau_{0}} \frac{\left(\beta_{2}\left(\tau(s), t_{1}\right)\right)^{1-\alpha}}{\beta_{1}\left(\tau(s), t_{1}\right) \tau^{\prime}(s)}\right)\right] d s=\infty
$$

then every solution of equation (1.1) is either oscillatory or tends to zero as $t \rightarrow \infty$.

Remark 3.1 From Theorem 3.1 to 3.4, we can get several oscillation criteria for equation (1.1) with different choices of the function $\rho$.

Next, we establish some Philos type oscillation results for equation (1.1).

\section{Definition 5.3.1[10]}

Consider the sets $\mathrm{D}_{0}=\left\{(t, s): t>s \geq t_{0}\right\}$ and $\mathrm{D}=\left\{(t, s): t \geq s \geq t_{0}\right\}$. Assume that $H \in C(\mathrm{D}, \mathrm{R})$ satisfies the following conditions:

(A1) $H(t, t)=0, t \geq t_{0} ; H(t, s)>0,(t, s) \in \mathrm{D}_{0} ;$

(A2) $H$ has a non-positive continuous partial derivative with respect to the second variable in $D_{0}$.

Then the function $H$ has the property $\mathrm{P}$.

Theorem 3.5 Let $\alpha \geq 1$. Assume condition (2.5) holds. Further assume that $H \in C(\mathrm{D}, \mathrm{R})$ has the property $\mathrm{P}$ and there exists a function $\rho \in C^{1}\left(\left[t_{0}, \infty\right),(0, \infty)\right)$ for all sufficiently large $t_{1} \geq t_{0}$, there is a $t_{2} \geq t_{1}$ such that

$$
-\frac{\partial}{\partial s} H(t, s)-\frac{\rho^{\prime}(s)}{\rho(s)} H(t, s)=\frac{h(t, s)(H(t, s))^{\frac{\alpha}{\alpha+1}}}{\rho(s)},(t, s) \in \mathrm{D}_{0}
$$

and

$$
\lim _{t \rightarrow \infty} \sup \frac{1}{H\left(t, t_{2}\right)} \int_{t_{2}}^{t} G_{1}(t, s) d s=\infty
$$

where

$$
G_{1}(t, s)=H(t, s) \frac{\rho(s) Q(s)}{2^{\alpha-1}}-\frac{\left(h_{-}(t, s)\right)^{\alpha+1}}{(\alpha+1)^{\alpha+1}}\left(\frac{1}{\left(\beta_{1}\left(s, t_{1}\right)\right)^{\alpha}}+\frac{p_{0}^{\alpha}}{\tau_{0}} \frac{1}{\left(\beta_{1}\left(\tau(s), t_{1}\right) \tau^{\prime}(s)\right)^{\alpha}}\right),
$$


$h_{-}(t, s)=\max \{0,-h(t, s)\}$. Then every solution of equation (1.1) is either oscillatory or tends to zero as $t \rightarrow \infty$.

Proof. Let $x(t)$ be a positive solution of equation (1.1). Then the corresponding function $z(t)$ satisfies Case $(I)$ and Case(II) of Lemma 2.3 for $t \geq t_{1}$.

Case(I). Define $w$ and $v$ as in the proof of Theorem 3.1. Then we obtain (3.8). Replace $t$ by $s$ and then multiply both sides of (??) by $H(t, s)$, integrate with respect to $s$ from $t_{2}\left(t_{2} \geq t_{1}\right)$ to $t$, we have

$$
\begin{gathered}
\int_{t_{2}}^{t} H(t, s) \frac{\rho(s) Q(s)}{2^{\alpha-1}} d s \leq-\int_{t_{2}}^{t} H(t, s) w^{\prime}(s) d s+\int_{t_{2}}^{t} H(t, s) \frac{\rho^{\prime}(s)}{\rho(s)} w^{\prime}(s) d s-\int_{t_{2}}^{t} H(t, s) \frac{\alpha \beta_{1}\left(s, t_{1}\right)}{\rho^{1 / \alpha}(s)} w^{1+\frac{1}{\alpha}}(s) d s \\
-\frac{p_{0}^{\alpha}}{\tau_{0}} \int_{t_{2}}^{t} H(t, s) v^{\prime}(s) d s+\frac{p_{0}^{\alpha}}{\tau_{0}} \int_{t_{2}}^{t} H(t, s) \frac{\rho^{\prime}(s)}{\rho(s)} v^{\prime}(s) d s \\
-\frac{p_{0}^{\alpha}}{\tau_{0}} \int_{t_{2}}^{t} H(t, s) \frac{\alpha \beta_{1}\left(\tau(s), t_{1}\right) \tau^{\prime}(s)}{\rho^{1 / \alpha}(s)} v^{1+\frac{1}{\alpha}}(s) d s .
\end{gathered}
$$

Then, we obtain

$$
\begin{aligned}
\int_{t_{2}}^{t} H(t, s) \frac{\rho(s) Q(s)}{2^{\alpha-1}} d s \leq H( & \left.t, t_{2}\right) w\left(t_{2}\right)-\int_{t_{2}}^{t}\left[-\frac{\partial}{\partial s} H(t, s)-\frac{\rho^{\prime}(s)}{\rho(s)} H(t, s)\right] w(s) d s \\
& -\int_{t_{2}}^{t} H(t, s) \frac{\alpha \beta_{1}\left(s, t_{1}\right)}{\rho^{1 / \alpha}(s)} w^{1+\frac{1}{\alpha}}(s) d s+\frac{p_{0}^{\alpha}}{\tau_{0}} H\left(t, t_{2}\right) v^{\prime}\left(t_{2}\right) \\
& -\frac{p_{0}^{\alpha}}{\tau_{0}} \int_{t_{2}}^{t}\left[-\frac{\partial}{\partial s} H(t, s)-\frac{\rho^{\prime}(s)}{\rho(s)} H(t, s)\right] v(s) d s \\
& -\frac{p_{0}^{\alpha}}{\tau_{0}} \int_{t_{2}}^{t} H(t, s) \frac{\alpha \beta_{1}\left(\tau(s), t_{1}\right) \tau^{\prime}(s)}{\rho^{1 / \alpha}(s)} v^{1+\frac{1}{\alpha}}(s) d s .
\end{aligned}
$$

Then

$$
\begin{aligned}
\int_{t_{2}}^{t} H(t, s) \frac{\rho(s) Q(s)}{2^{\alpha-1}} d s \leq H\left(t, t_{2}\right) w\left(t_{2}\right) & +\frac{p_{0}^{\alpha}}{\tau_{0}} H\left(t, t_{2}\right) v^{\prime}\left(t_{2}\right) \\
& +\int_{t_{2}}^{t}\left[\frac{h_{-}(t, s)(H(t, s))^{\frac{\alpha}{\alpha+1}}}{\rho(s)} w(s)-H(t, s) \frac{\alpha \beta_{1}\left(s, t_{1}\right)}{\rho^{1 / \alpha}(s)} w^{1+\frac{1}{\alpha}}(s)\right] d s \\
& +\frac{p_{0}^{\alpha}}{\tau_{0}} \int_{t_{2}}^{t}\left[\frac{h_{-}(t, s)(H(t, s))^{\frac{\alpha}{\alpha+1}}}{\rho(s)} v(s)-H(t, s) \frac{\alpha \beta_{1}\left(\tau(s), t_{1}\right) \tau^{\prime}(s)}{\rho^{1 / \alpha}(s)} v^{1+\frac{1}{\alpha}}(s)\right] d s
\end{aligned}
$$

using the inequality (3.9) in the last inequality, we obtain

$\frac{1}{H\left(t, t_{1}\right)} \int_{t_{2}}^{t}\left[H(t, s) \frac{\rho(s) Q(s)}{2^{\alpha-1}}-\frac{\left(h_{-}(t, s)\right)^{\alpha+1}}{(\alpha+1)^{\alpha+1} \rho^{\alpha}(s)}\left(\frac{1}{\left(\beta_{1}\left(s, t_{1}\right)\right)^{\alpha}}+\frac{1}{\left(\beta_{1}\left(\tau(s), t_{1}\right) \tau^{\prime}(s)\right)^{\alpha}}\right)\right] d s \leq w\left(t_{2}\right)+\frac{p_{0}^{\alpha}}{\tau_{0}} v\left(t_{2}\right)$, 
which contradicts (3.20).

Case (II). The proof is similar to that of Theorem 3.1. The proof is now complete.

From Theorem 3.2, similar to that proof of Theorem 3.5, we derive the following theorem.

Theorem 3.6 Let $0<\alpha \leq 1$. Assume that condition (2.5) holds. Moreover assume that $H \in C(\mathrm{D}, \mathrm{R})$ has the property $\mathrm{P}$, and there exists a function $\rho \in C^{1}\left(\left[t_{0}, \infty\right),(0, \infty)\right)$ for all sufficiently large $t_{1} \geq t_{0}$, there is a $t_{2} \geq t_{1}$ such that (3.19) holds, and

$$
\lim _{t \rightarrow \infty} \sup \frac{1}{H\left(t, t_{2}\right)} \int_{t_{2}}^{t} F_{1}(t, s) d s=\infty
$$

where

$$
F_{1}(t, s)=H(t, s) \rho(s) Q(s)-\frac{\left(h_{-}(t, s)\right)^{\alpha+1}}{(\alpha+1)^{\alpha+1} \rho^{\alpha}(s)}\left(\frac{1}{\left(\beta_{1}\left(s, t_{1}\right)\right)^{\alpha}}+\frac{1}{\left(\beta_{1}\left(\tau(s), t_{1}\right) \tau^{\prime}(s)\right)^{\alpha}}\right)
$$

$h_{-}(t, s)=\max \{0,-h(t, s)\}$. Then every solution of equation (1.1) is either oscillatory or tends to zero as $t \rightarrow \infty$.

From (3.17) in Theorem 3.3, similar to that proof of Theorem 3.5, we obtain the following results.

Theorem 3.7 Let $\alpha \geq 1$. Assume that condition (2.5) holds. Further, assume that $H \in C(\mathrm{D}, \mathrm{R})$ has the property $\mathrm{P}$, and there exists a function $\rho \in C^{1}\left(\left[t_{0}, \infty\right),(0, \infty)\right)$, for all sufficiently large $t_{1} \geq t_{0}$, there is a $t_{2}>t_{1}$ such that

$$
-\frac{\partial}{\partial s} H(t, s)-\frac{\rho^{\prime}(s)}{\rho(s)} H(t, s)=\frac{h(t, s)(H(t, s))^{1 / 2}}{\rho(s)},(t, s) \in \mathrm{D}_{0},
$$

and

$$
\lim _{t \rightarrow \infty} \sup \frac{1}{H\left(t, t_{2}\right)} \int_{t_{2}}^{t} G_{2}(t, s) d s=\infty
$$

where

$$
G_{2}(t, s)=H(t, s) \frac{\rho(s) Q(s)}{2^{\alpha-1}}-\frac{\left(h_{-}(t, s)\right)^{2}}{4 \alpha \rho(s)}\left(\frac{\left(\beta_{2}\left(s, t_{1}\right)\right)^{1-\alpha}}{\beta_{1}\left(s, t_{1}\right)}+\frac{p_{0}^{\alpha}}{\tau_{0}} \frac{\left(\beta_{2}\left(\tau(s), t_{1}\right)\right)^{1-\alpha}}{\beta_{1}\left(\tau(s), t_{1}\right) \tau^{\prime}(s)}\right)
$$

$h_{-}(t, s)=\max \{0,-h(t, s)\}$. Then every solution of equation (1.1) is either oscillatory or tends to zero as $t \rightarrow \infty$.

By Theorem 3.5, similar to the proof of Theorem 3.5, we establish the following criteria.

Theorem 3.8 Let $0<\alpha \leq 1$. Assume that condition (2.5) holds. Further, assume that $H \in C(\mathrm{D}, \mathrm{R})$ has the property $\mathrm{P}$, and there exists a function $\rho \in C^{1}\left(\left[t_{0}, \infty\right),(0, \infty)\right)$, for all sufficiently large $t_{1} \geq t_{0}$, there is a $t_{2}>t_{1}$ such that (3.22) holds and

$$
\lim _{t \rightarrow \infty} \sup \int_{t_{1}}^{t} F_{2}(t, s) d s=\infty
$$

where

$$
F_{2}(t, s)=H(t, s) \rho(s) Q(s)-\frac{\left(h_{-}(t, s)\right)^{2}}{4 \alpha \rho(s)}\left(\frac{\left(\beta_{2}\left(s, t_{1}\right)\right)^{1-\alpha}}{\beta_{1}\left(s, t_{1}\right)}+\frac{p_{0}^{\alpha}}{\tau_{0}} \frac{\left(\beta_{2}\left(\tau(s), t_{1}\right)\right)^{1-\alpha}}{\beta_{1}\left(\tau(s), t_{1}\right) \tau^{\prime}(s)}\right),
$$

$h_{-}(t, s)=\max \{0,-h(t, s)\}$. Then every solution of equation (1.1) is either oscillatory or tends to zero as $t \rightarrow \infty$.

Remark 3.2 From Theorem 3.5-3.8, we can obtain several oscillation criteria for equation (1.1) with different choices of function $\rho$ and $H$. 


\section{Examples}

In this section, we will present three examples to illustrate the main results.

Example 4.1 Consider the third order half-linear neutral differential equation with "maxima"

$$
\left[t\left(\left(x(t)+p_{0} x(t / 2)\right)^{\prime \prime}\right)^{3}\right]+\frac{\lambda}{t^{6}} \max _{[t / 2, t]} x^{3}(s)=0, t \geq 1,
$$

where $\lambda>0$. Here $a(t)=t, p(t)=p_{0}>0, \tau(t)=\sigma(t)=t / 2, \alpha=3, q(t)=\frac{\lambda}{t^{6}}$, and $\tau_{0}=\frac{1}{2}$. Then $Q(t)=\frac{\lambda}{t^{6}}$, and

$$
\beta_{1}\left(t, t_{1}\right)=\int_{t_{1}}^{t} \frac{1}{a^{1 / \alpha}(s)} d s=\int_{t_{1}}^{t} \frac{1}{s^{1 / 3}} d s \geq t^{2 / 3}
$$

for $t$ sufficiently large. It is easy to see that condition (2.5) holds. Set $\rho(t)=t^{5}$. We obtain

$$
\begin{gathered}
\lim _{t \rightarrow \infty} \sup \int_{t_{2}}^{t}\left[\frac{\rho(s) Q(s)}{2^{\alpha-1}}-\frac{\left(\rho^{\prime}(s)\right)^{\alpha+1}}{(\alpha+1)^{\alpha+1}(\rho(s))^{\alpha}}\left(\frac{1}{\left(\beta_{1}\left(s, t_{1}\right)\right)^{\alpha}}+\frac{\frac{p_{0}^{\alpha}}{\tau_{0}}}{\left(\beta_{1}\left(\tau(s), t_{1}\right) \tau^{\prime}(s)\right)^{\alpha}}\right] d s\right. \\
\geq\left[\frac{\lambda}{4}-\frac{5^{4}\left(1+64 p_{0}^{3}\right)}{4^{4}}\right] \lim _{t \rightarrow \infty} \sup \int_{t_{2}}^{t} \frac{1}{s} d s=\infty
\end{gathered}
$$

provided $\lambda>\frac{5^{4}\left(1+64 p_{0}^{3}\right)}{4^{3}}$. Hence by Theorem 3.1, every solution of equation (4.1) is either oscillatory or tends to zero as $t \rightarrow \infty$ when $\lambda>\frac{5^{4}\left(1+64 p_{2}^{3}\right)}{4^{3}}$.

Example 4.2 Consider the third order neutral differential equation with "maxima"

$$
\left(t\left((x(t)+2 x(t / 2))^{\prime \prime}\right)^{3}\right)+\frac{\lambda}{t^{6}} \max _{[t / 2, t]} x^{3}(s)=0, t \geq 1,
$$

where $\lambda>0$. Here $a(t)=t, p(t)=p_{0}>0, \tau(t)=\sigma(t)=t / 2, \alpha=3, q(t)=\frac{\lambda}{t^{6}}$, and $\tau_{0}=\frac{1}{2}$. Then $Q(t)=\frac{\lambda}{t^{6}}$, and $\beta_{1}\left(t, t_{1}\right) \geq t^{2 / 3}$ and $\beta_{2}\left(t, t_{1}\right) \geq \frac{2}{5} t^{5 / 3}$. Clearly condition (2.5) holds. Set $\rho(t)=t^{5}$. We obtain $\lim _{t \rightarrow \infty} \sup \int_{t_{2}}^{t}\left[\frac{\rho(s) Q(s)}{2^{\alpha-1}}-\frac{\left(\rho^{\prime}(s)\right)^{2}}{4 \alpha \rho(s)}\left(\frac{\left(\beta_{2}\left(s, t_{1}\right)\right)^{1-\alpha}}{\beta_{1}\left(s, t_{1}\right)}+\frac{p_{0}^{\alpha}}{\tau_{0}} \frac{\left(\beta_{2}\left(\tau(s), t_{1}\right)\right)^{1-\alpha}}{\beta_{1}\left(s, t_{1}\right) \tau^{\prime}(s)}\right)\right] d s \geq\left[\frac{\lambda}{4}-\frac{5^{4}\left(1+2^{6} p_{0}^{3}\right)}{48}\right] \lim _{t \rightarrow \infty} \sup \int_{t_{2}}^{t} \frac{1}{S} d s=\infty$,

provided $\lambda>\frac{5^{4}\left(1+2^{6} p_{0}^{3}\right)}{12}$. Hence by Theorem 3.3, every solution of equation (4.2) is either oscillatory or tends to zero as $t \rightarrow \infty$.

Example 4.3 Consider the third order half-linear neutral differential equation with "maxima"

$$
\left(t^{1 / 9}\left(\left(x(t)+p_{0} x(t / 2)\right)^{\prime \prime}\right)^{1 / 3}\right)+\frac{\lambda}{t^{14 / 9}} \max _{[t / 2, t]} x^{1 / 3}(s)=0, t \geq 1,
$$


where $\lambda>0$. Here $a(t)=t^{1 / 9}, p(t)=p_{0}>0, \tau(t)=\sigma(t)=t / 2, \alpha=1 / 3, q(t)=\frac{\lambda}{t^{14 / 9}}$, and $\tau_{0}=\frac{1}{2}$. Then $Q(t)=\frac{\lambda}{t^{14 / 9}}$, and $\beta_{1}\left(t, t_{1}\right) \geq t^{2 / 3}$ for $t$ sufficiently large. It is easy to see that conditio (2.5) holds. Set $\rho(t)=t^{4 / 3}$. We obtain

$\lim _{t \rightarrow \infty} \sup \int_{t_{2}}^{t}\left[\rho(s) Q(s)-\frac{\left(\rho^{\prime}(s)\right)^{\alpha+1}}{(\alpha+1)^{\alpha+1} \rho^{\alpha}(s)}\left(\frac{1}{\left(\beta_{1}\left(s, t_{1}\right)\right)^{\alpha}}+\frac{\frac{p_{0}^{\alpha}}{\tau_{0}}}{\left(\beta_{1}\left(\tau(s), t_{1}\right) \tau^{\prime}(s)\right)^{\alpha}}\right)\right] d s \geq\left[\lambda-\left(1+2^{5 / 3} p_{0}^{1 / 3}\right)\right] \lim _{t \rightarrow \infty} \sup \int_{t_{2}}^{t} \frac{1}{s^{2 / 9}} d s=\infty$,

if $\lambda>\left(1+2^{5 / 3} p_{0}^{1 / 3}\right)$. Hence by Theorem 3.2, every solution of equation (4.3) is either oscillatory or tends to zero as $t \rightarrow \infty$. We conclude this paper with the following remark.

Remark 4.1 In this paper, we have established some new oscillation theorems for the equation (1.1) for the case $0 \leq p(t) \leq p_{0}<\infty$. It would be interesting to investigate equation (1.1) under the case when $p(t)<-1$, $\lim _{t \rightarrow \infty} p(t)=\infty$ or $p(t)$ is an oscillatory function. Further it is interesting to find different method to remove the condition $\sigma \circ \tau=\tau \circ \sigma$.

\section{References}

[1] R.P.Agarwal, M.F.Aktas and A.Tiryaki, On oscillation criteria for third order nonlinear delay differential equations, Arch. Math. (Brno). 45(2009), 1-18.

[2] R.Arul and M.Mani, On the oscillation of third order quasilinear delay differential equations with "maxima", Malaya J. Mathematik, 2 (2014), 489-496.

[3] R.Arul and M.Mani, Oscillation of third order nonlinear delay differential equation with "maxima", Communications in Differential and Difference Equations, 5 (2014), 25-34.

[4] R.Arul and M.Mani, Oscillation solution to third order half-linear neutal differential equation with "maxima", Far East J. Math. Sci., 90 (2014), 89-101.

[5] R.Arul and A.Ashok, Oscillation Theorems for Third Order Nonlinear Delay Differential Equation with "Maxima", J. Prog. Research Math., 12(1) (2017), 1739-1747.

[6] R.Arul and A.Ashok, Oscillation and asymptotic behavior of third order half-linear delay differential equation with "Maxima", Far East J. Appl. Math., (to appear).

[7] B.Baculikova and J.Dzurina, Oscillation of third order functional differential equations, Electron. J. Qual. Theory Differ. Equ., 43 (2010), 1-10.

[8] B.Baculikova and J.Dzurina, On the asymptotic behavior of a class of third order nonlinear neutral differential equations, Cent. Eur. J. Math., 8 (2010), 1091-1103.

[9] B.Karpuz, O.Ocalan and S.Ozturk, Comparison theorems on the oscillation and asymptotic behavior of higher order neutral differential equations, Glasgow Math. J., 52 (2010), 107-114.

[10] Ch.G.Philos, Oscillation theorems for linear differential equations of second order, Arch. Math., 53, (1989), 482-492.

[11] Richard R.Goldberg, Methods of Real Analysis, Oxford and IBH Publishing Comp., New Delhi, 1970.

[12] M.Su and Z.Xu, Oscillation criteria of certain third order neutral differential equations, Differ. Equ. Appl., 4(2012), 221-232.

[13] E.Thandapani and T.Li, On the oscillation of third order quasilinear neutral functional differential equations, Arch. Math. (Brno), 47 (2011), 181-199.

[14] E.Thandapani, S.Tamilvanan and E.S.Jambulingam, Oscillatory behavior of third order nonlinear neutral differential equations, Inter. J. Pure Appl. Math., 94 (2014), 55-63.

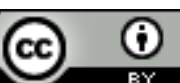

This work is licensed under a Creative Commons Attribution 4.0 International License. 